\title{
RECUPERACIÓN DE COBRE CONTENIDO EN ESCORIA DE COBRE MEDIANTE FLOTACIÓN
}

\author{
L. VALDERRAMA ${ }^{1 *}$, M. GONZÁLEZ ${ }^{1,2}$, M. SANTANDER ${ }^{2}$, B. ZAZZALI ${ }^{1}$ \\ ${ }^{1}$ Departamento de Ingeniería en Metalurgia, Universidad de Atacama, Copiapó, Chile, ${ }^{2}$ Planta Manuel Antonio \\ Matta, Empresa Nacional de Minería, Copiapó, Chile \\ luis.valderrama@uda.cl
}

Recepción 21/03/2018 - Aprobacíon 22/10/2018

DOI: $10.15628 /$ holos. 2018.7118

\section{RESUMEN}

El cobre se obtiene principalmente a partir de los sulfuros de cobre, mediante procesos clásicos de flotación y pirometalúrgicos, siendo Chile el mayor productor de cobre en el mundo. En nuestro país existen siete fundiciones de cobre que generan grandes volúmenes de escoria. Este trabajo presenta los resultados obtenidos de la flotación de cobre de escorias del horno de reverbero (HR) y del convertidor Teniente (CT) de la Fundición Hernán Videla Lira Paipote. Así, mediante pruebas de flotación rougher, a escala de laboratorio, se determinó que la granulometría, para ambos casos, corresponde a $70 \%$ menor a $74 \mu \mathrm{m}$, lográndose recuperaciones de 59,2\% escoria HR y $86,8 \%$ para la escoria del CT, cuyas leyes de cobre en el concentrado fueron de $3,7 \%$ y $23,6 \%$ respectivamente. Las dosis de reactivos utilizados fueron 40 g/t de F-703 como colector y $30 \mathrm{~g} / \mathrm{t}$ de $\mathrm{H}-70$ como espumante. Los tiempos óptimos de flotación de las etapas rougher y cleaner, de ambas escorias, se determinaron mediante cinéticas de flotación. Finalmente se realizaron pruebas a nivel industrial con los resultados de laboratorio, alcanzándose una ley de $18,2 \%$ de cobre (recuperación de $56,1 \%$ ) para escoria $\mathrm{HR}$, mientras que para la escoria CT se obtuvo una ley de $39,1 \%$ de cobre con una recuperación de $91,4 \%$

PALABRAS-CLAVE: escoria de cobre, recuperación, flotación.

\section{RECOVERY OF COPPER CONTAINED IN COPPER SLAG THROUGH FLOTATION}

\begin{abstract}
Copper is obtained mainly from copper sulphides, through classical flotation and pyrometallurgical processes, with Chile being the largest producer of copper in the world. In our country there are seven copper smelters that generate large volumes of slag. This paper presents the results obtained from the flotation of copper slag from the reverberatory furnace $(\mathrm{HR})$ and the Teniente converter (CT) from the Hernán Videla Lira - Paipote smelter. Thus, by rougher flotation tests, at laboratory scale, it was determined that the granulometry, for both cases, corresponds to $70 \%$ less than $74 \mu \mathrm{m}$, achieving recoveries of $59.2 \% \mathrm{HR}$ slag and
\end{abstract}

$86.8 \%$ for the slag of the $\mathrm{CT}$, whose copper grades in the concentrate were $3.7 \%$ and $23.6 \%$ respectively. The doses of reagents used were $40 \mathrm{~g} / \mathrm{t}$ of $\mathrm{F}-703$ as a collector and $30 \mathrm{~g} / \mathrm{t}$ of $\mathrm{H}-70$ as foaming agent. The optimal flotation times of the rougher and cleaner stages of both slags were determined by flotation kinetics. Finally, industrial tests were carried out with laboratory results, reaching a degree of $18.2 \%$ copper (recovery of $56.1 \%$ ) for HR slag, while for CT slag a degree of $39.1 \%$ was obtained. of copper with a recovery of $91.4 \%$ 


\section{INTRODUCCIÓN}

La obtención de cobre por procesos pirometalúrgicos a nivel mundial alcanza alrededor del 80\% (Davenport, et al., 2002). Por cada tonelada de cobre se generan entre 2,0 a 3,0 toneladas de escoria, dependiendo de las propiedades de los concentrados de cobre y las condiciones de operación en el proceso pirometalúrgico (Gorai, et al., 2003). Una desventaja de tal proceso es que las escorias de cobre no se utilizan de manera efectiva para recuperar las especies de valor contenidas en ellas, sino que, por el contrario, causan graves daños al medio ambiente, contaminando con metales pesados los suelos y cursos de agua circundantes (Antonijevica, et al., 2008).

El proceso pirometalúrgico de una fundición busca eliminar la mayor cantidad de hierro, azufre y ganga, minimizando la pérdida de cobre en las escorias. Esto se logra mediante la adición de sílice, de modo que la escoria fayalítica formada capture al hierro; mientras que el aire insuflado a alta temperatura elimina el azufre como dióxido de azufre, (Hayes, 1993).

Las escorias de cobre, que generalmente contienen una gran cantidad de metales valiosos, en la actualidad son considerados recursos secundarios de metales. El tratamiento de tales escorias mediante procesos de chancado, molienda, separación magnética, flotación, lixiviación y tostación, permite recuperar metales tales como $\mathrm{Fe}, \mathrm{Cr}, \mathrm{Cu}, \mathrm{Al}, \mathrm{Pb}, \mathrm{Zn}, \mathrm{Co}, \mathrm{Ni}, \mathrm{Nb}, \mathrm{Ta}$, Au y Ag. En ese mismo sentido, la recuperación de metales de las escorias es importante, no solo para ahorrar recursos de metal, sino que también permite proteger el medio ambiente (González et al., 2005; Shen y Forssberg, 2003; Kim et al., 2013).

Estudios científicos han mostrado que es importante realizar un adecuado método de enfriamiento de la escoria, lo que impacta significativamente en la recuperación de cobre en el proceso de flotación. Como consecuencia de lo anterior, tal procedimiento tecnológico proporciona mejores resultados porque promueve el crecimiento o la coagulación de partículas dispersas de cobre sulfurado y metálico en la escoria, aumentando la recuperación del proceso de flotación con la disminución de pérdidas de cobre a través de partículas muy finas (Alexandrova, et al., 2014; Mihajlović, et al., 2015).

Actualmente en Chile existen siete fundiciones de cobre, de las cuales cinco son estatales y dos privadas. De la fundiciones estatales cuatro pertenecen a CODELCO: Chuquicamata, Potrerillos, Ventanas y Caletones, y una a ENAMI: la fundición Hernán Videla Lira, de Paipote. Las dos restantes son Altonorte y Chagres. En Caletones, Potrerillos y Chuquicamata están reemplazando los hornos de tratamiento de escorias por procesos de molienda y flotación. (Minería Chilena 2015; Portal Minero, 2017).

En general, las pérdidas de cobre en las escorias de reverbero pueden ser químicas o mecánicas, siendo las pérdidas mecánicas las más importantes, principalmente en forma de gotas (Biswas y Davenport, 1980). Entre los factores principales que afectan las pérdidas mecánicas se encuentran: la viscosidad de la escoria, las propiedades interfaciales, la turbulencia del baño y las composiciones químicas de la escoria (Ip y Toguri, 1992).

Asimismo, han sido informados varios métodos para la recuperación de cobre de las escorias, entre los que se puede mencionar los procesos pirometalúrgicos, hidrometalúrgicos y procesos mixtos. Sin embargo, la separación por flotación ha sido ampliamente aplicada y 
algunos investigadores la consideran como un método efectivo para recuperar el cobre contenido en las escorias (Roy, et al., 2015; Altundogan y Tumen, 1997; Mehta et al., 1999; Herreros et al., 1998).

Del mismo modo, la hidrofobicidad del cobre se puede mejorar mediante la adición de colectores genéricos como xantato y ditiofosfatos, los cuales también son efectivos en la flotación de sulfuro de cobre presentes en las escorias. Así, los xantatos y ditiofosfatos son típicos colectores tiol que forman diferentes productos en la superficie de los sulfuro de cobre. Por ende, se ha demostrado que el uso de mezclas de colectores tiol puede tener beneficios con respecto al uso de colectores puros (Bradshaw y O'Connor, 1996; Bradshaw et al., 1998; Rao y Forrsberg, 1997).

En consecuencia, la flotación es técnica y económicamente superior a otros procesos empleados para la recuperación de cobre contenido en las escorias (Rao y Nayak, 1992; Piret, 2000; Somerville et al., 2000). De igual manera, otras técnicas que han sido estudiadas técnica y económicamente, son el enfriamiento gradual de la escoria (Sarrafi et al., 2004) y la adición de pirita para incrementar la fluidez de la escoria y así producir un menor tamaño de las gotas de cobre atrapado, generando a la vez un aumento de la recuperación y ley en concentrado en la flotación (Bulut et al., 2006; Demetrio et al., 2000).

En base a las consideraciones anteriores, en el presente trabajo se investiga el procesamiento de las escorias de reverbero y convertidor Teniente producidas en la Fundición Hernán Videla Lira, mediante flotación, para recuperar el cobre contenido. En tal sentido, fueron realizadas la caracterización química, mineralógica y metalúrgica de ambas escoria, luego se realizaron pruebas de flotación para determinar las variables de operación para las etapas rougher y cleaner usando como colector Hostaflot E-703 y como espumante Flotanol H-70, para ambos tipos de escoria.

\section{MATERIALES Y MÉTODOS}

\subsection{Materiales}

En este trabajo se utilizaron escorias de cobre, provenientes de la operación del horno reverbero y del Convertidor Teniente, ambos de la Fundición Hernán Videla Lira de la ENAMI Chile. Para extraer las muestras se diseñó una malla de muestreo en el escorial y estas fueron tomadas mediante calicatas usando una retroexcavadora, se tomaron aproximadamente 30 toneladas de cada escorial, las que fueron reducidas de tamaño hasta $1,8 \mathrm{~mm}$. Mediante cuarteos sucesivos se tomó una sub muestra de 200 kilogramos, para su caracterización cinéticas de molienda y pruebas de flotación de laboratorios; el resto para la prueba industrial.

La composición química fue realizada por análisis convencionales, el peso específico se determinó por el método del picnómetro, mientras que la mineralogía y fases cristalinas se analizaron por QEMSCAN.

Los parámetros metalúrgicos y los reactivos estudiados en las pruebas realizadas en este trabajo, fueron usados en base a los empleados en Fundición Potrerillos de Codelco Chile División El Salvador y por antecedentes bibliográficos de otras fundiciones que recuperan cobre contenido en la escoria por flotación. 


\subsection{Determinación del índice de Bond y abrasión}

El índice de Bond se determinó mediante un molino de bola estándar de laboratorio de $30,5 \mathrm{~cm}$ de diámetro $\times 30,5$ de largo, que gira a $70 \mathrm{rpm}$, conteniendo una carga de bolas que va de $3,8 \mathrm{~cm}$ a 1,9 cm, con una carga total de 285 bolas, utilizándose 125,0 $\mu \mathrm{m}$ como malla de corte. Del mismo modo, el índice de abrasión se determinó mediante un molino con 400 gramos de escoria que giraba a $70 \mathrm{rpm}$. Al finalizar la prueba se retiró y limpió con prolijidad la paleta, y luego se pesó en una balanza de precisión (cuatro dígitos). Finalizada la prueba, después de cuatro mediciones, se grafica la pérdida de masa acumulada en función del tiempo.

\subsection{Pruebas de molienda}

Las pruebas de molienda en húmedo se efectuaron para determinar los tiempos en que se alcanzaban granulometrías de $65 \%, 70 \%$ y $75 \%$ bajo 75 micrómetros; similarmente, estos ensayos se realizaron en un molino de bolas marca Hebro, de acero inoxidable de $23,5 \mathrm{~cm}$ de largo por $17,5 \mathrm{~cm}$ de diámetro; con una carga de bolas de 10 kilogramos y un porcentaje de sólidos de $67 \%$ $(\mathrm{p} / \mathrm{p})$.

\subsection{Pruebas de flotación}

Las pruebas de flotación de laboratorio se llevaron a cabo en una celda marca Denver D12, utilizando una muestra de escoria molida. Operacionalmente la velocidad del impulsor fue constante (1.300 rpm). La prueba industrial se realizó en planta M. A. Matta, en una sección que está constituido por un molino bolas, con descarga por rebalse y su clasificación mediante ciclones Krebs. El circuito de flotación se compone de 1 celdas OK-8 de $8 \mathrm{~m}^{3}$ de capacidad para la etapa rougher; 8 celdas OK- 8 , de $8 \mathrm{~m}^{3}$ de capacidad cada una, para la etapa scavenger. 1 celdas OK-3, de $2,8 \mathrm{~m}^{3}$ de capacidad para la etapa cleaner. Todas las pruebas de flotación fueron realizadas a $\mathrm{pH} 8$, con los reactivos de la Clariant, Hostaflot E-703 (tiono carbamato modificado) como colector y Flotanol H-70 (mezcla de éteres poliglicólicos y alcoholes) como espumante.

\section{RESULTADOS}

\subsection{Análisis químico}

La tabla 1 muestra la composición química de los principales elementos y compuestos presentes en las escorias.

Tabla 1. Análisis de químico de las escorias en porcentaje

\begin{tabular}{lccccccccc}
\hline Escoria & $\mathrm{Cu}$ & $\mathrm{Fe}$ & $\mathrm{S}$ & $\mathrm{SiO}_{2}$ & $\mathrm{CaO}$ & $\mathrm{MgO}$ & $\mathrm{Al}_{2} \mathrm{O}_{3}$ & $\mathrm{Na}_{2} \mathrm{O}$ & $\mathrm{K}$ \\
H. Reverbero (HR) & 0,83 & 33,3 & 0,5 & 37,9 & 7,7 & 1,4 & 7,5 & 2,1 & 1,1 \\
C. Teniente (CT) & 4,56 & 40,3 & 1,53 & 20,3 & 0,33 & 0,75 & 2,86 & 1,2 & 0,8 \\
\hline
\end{tabular}

El peso específico de la escoria del HR es $3,3 \mathrm{~kg} / \mathrm{L}$ y la del CT es $4,1 \mathrm{~kg} / \mathrm{L}$.

En las figuras 1 y 2 se muestran los difractogramas de la escoria del HR y CT, en los que se observa que las principales fases cristalinas de la escoria son fayalita $\left(\mathrm{Fe}_{2} \mathrm{SiO}_{4}\right)$, magnetita $\left(\mathrm{Fe}_{3} \mathrm{O}_{4}\right)$, cuarzo $\left(\mathrm{SiO}_{2}\right)$ y algunos sulfuros de hierro y cobre. 


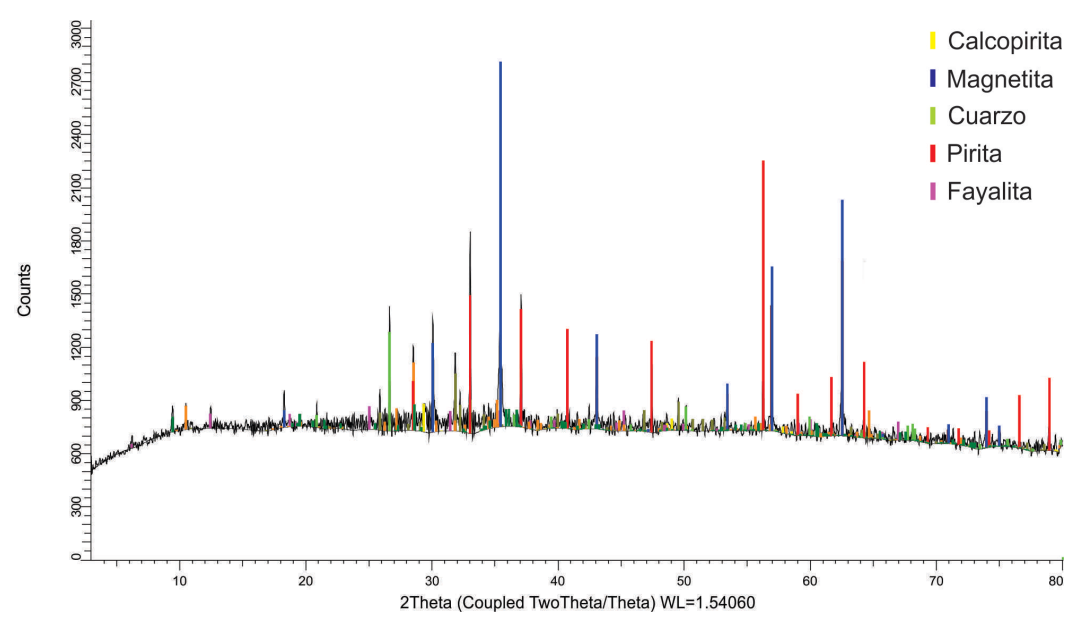

Figura 1. Difracción de rayos $\mathrm{X}$ de la escoria de reverbero

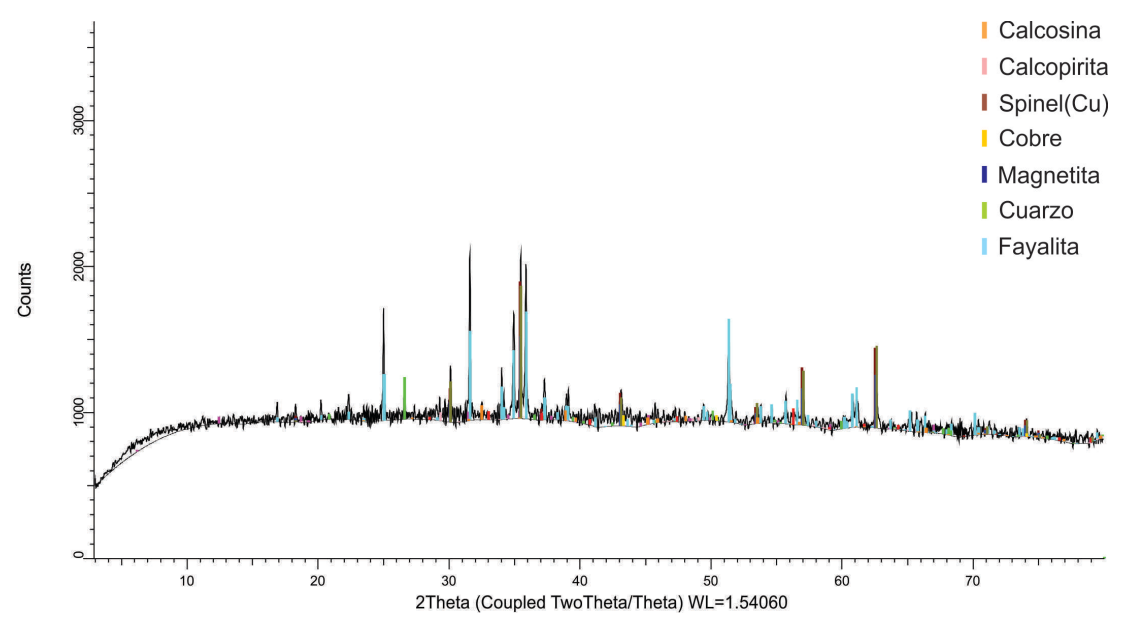

Figura 2. Difracción de rayos $X$ de la escoria de convertidor Teniente

\subsection{Determinación del Índice de Bond y de abrasión}

El Índice de Bond (Wi) para la escoria del HR alcanzó un valor de 23,7 KWh/t, mientras que la escoria del CT entregó un valor de 23,4 KWh/t. De igual manera, el Índice de Abrasión para la escoria del HR fue de $0,9119 \mathrm{~g} / \mathrm{h}$ y para la escoria del CT fue de $0,7161 \mathrm{~g} / \mathrm{h}$.

\subsection{Pruebas de molienda}

Las pruebas de molienda se realizan para determinar la granulometría óptima para la flotación. Así, se ejecutaron pruebas de molienda para establecer los tiempos requeridos para tener granulometrías de $65 \%, 70 \%$ y $75 \%$ bajo 75 micrómetros. De esta manera, se determinó que el tiempo necesario para obtener tamaños de $70 \%$ bajo los 75 micrómetros para la escoria del HR fue de 32,1 minutos, mientras que para la escoria del CT esta granulometría se consigue a los 31,9 minutos.

\subsection{Pruebas de flotación}

Las pruebas de flotación, para ambas escorias, se realizaron con las granulometrías reportadas en la pruebas de molienda, usando dosis de $40 \mathrm{~g} / \mathrm{t}$ para el colector (E-703) y de espumante $30 \mathrm{~g} / \mathrm{t}$ (H-70), cuyos tiempos de flotación fueron 15 minutos y un porcentaje de sólido de $35 \%(\mathrm{p} / \mathrm{p})$ y $\mathrm{pH}$. Los resultados de tales pruebas se presentan en las figuras 3 y 4 , 
donde se observa el efecto de la granulometría en la recuperación y ley de cobre en el concentrado.

Esas mismas pruebas indican que un aumento en la fineza de la escoria HR no garantiza un efecto significativo en la recuperación o en la ley del concentrado obtenido, donde las recuperaciones no superan el $60 \%$ con leyes de cobre en el concentrado por debajo del $5 \%$; como consecuencia de ello, se hace necesario una etapa de limpieza para lograr una mejor calidad del concentrado, tal como se observa en la Figura 3. Por otra parte, el mejor resultado se alcanza con una granulometría de $70 \%$ menor a 75 micrómetros, con una recuperación de $62,2 \%$ y ley de cobre en el concentrado de $3,7 \%$. Esta baja recuperación podría deberse a la baja ley de alimentación, así como el estado químico de cobre presente en este material. La figura también indica que una menor granulometría no significa una mayor recuperación, posiblemente debido a una mayor cantidad de lamas, y estas podrían producir el recubrimiento de las partículas hidrofóbicas por estos ultrafinos (Ip y Toguri, 1992).

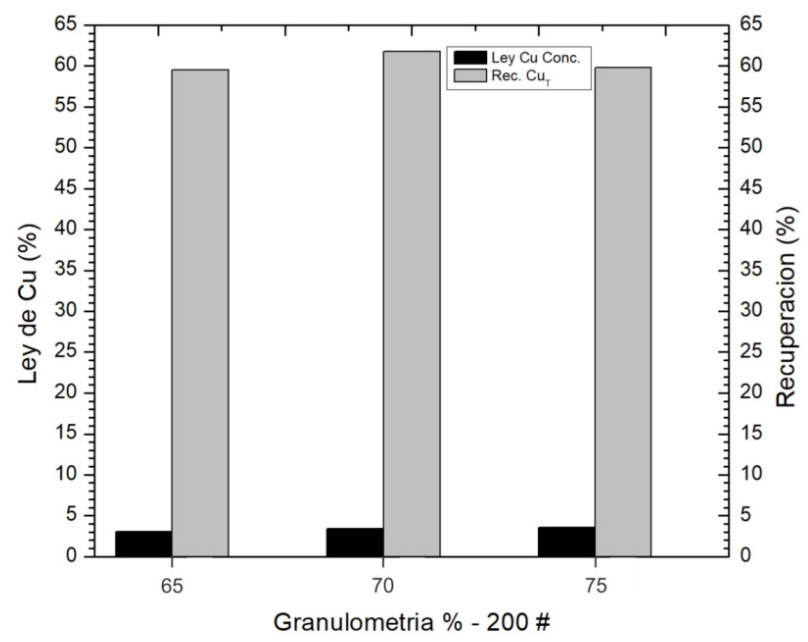

Figura 3. Efecto de la granulometría sobre la ley y recuperación de cobre en escoria HR.

Las escorias provenientes del CT tienen una ley de cobre en la alimentación superior a 4\%, condición que se observa en la Figura 4, lo que favorece la ley de cobre en el concentrado, superando el $20 \%$, con recuperaciones levemente inferiores a $90 \%$. Tal como se establece en la Figura 4, en todas las condiciones granulométricas consideradas, para una granulometría $70 \%$ bajo los 75 micrómetros, se logra una recuperación de $86,8 \%$ con una ley de cobre en el concentrado de $23,6 \%$. 


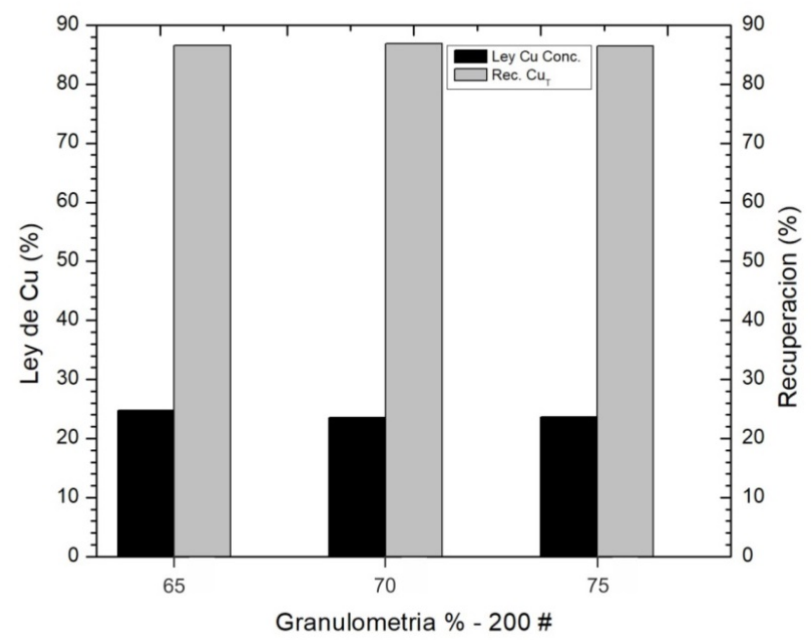

Figura 4. Efecto de la granulometría sobre la ley y recuperación de cobre de escoria del CT.

\subsection{Cinética de flotación}

Considerando los resultados de las pruebas de flotación rougher respecto del efecto de la granulometría, se procedió posteriormente a realizar cinéticas de flotación (rougher y cleaner) para determinar el tiempo óptimo. De esta manera, las condiciones experimentales fueron: granulometría $70 \%$ bajo $75 \mu \mathrm{m}, \mathrm{pH} 8,40 \mathrm{~g} / \mathrm{t}$ de colector (F-703) y $30 \mathrm{~g} / \mathrm{t}$ de espumante (H -70).

En la Figura 5 (a) se presenta la cinética de flotación rougher realizada a la escoria HR. En base a las condiciones experimentales, se observa que en los primeros tres minutos la ley de cobre en el concentrado es $5,7 \%$, con recuperaciones que alcanzan a valores de $45,7 \%$. Si bien esta recuperación es más baja que la obtenida en pruebas preliminares, se resalta que el concentrado es de mejor calidad, puesto que, al aumentar el tiempo de flotación, tanto en la ley como en la recuperación sucederán cambios. Así, de acuerdo al criterio de Agar et al., (1980), se deduce que el tiempo óptimo de flotación es de 6 minutos 12 segundos, con una ley de cobre de $3,7 \%$ de cobre y una recuperación de $62,1 \%$.
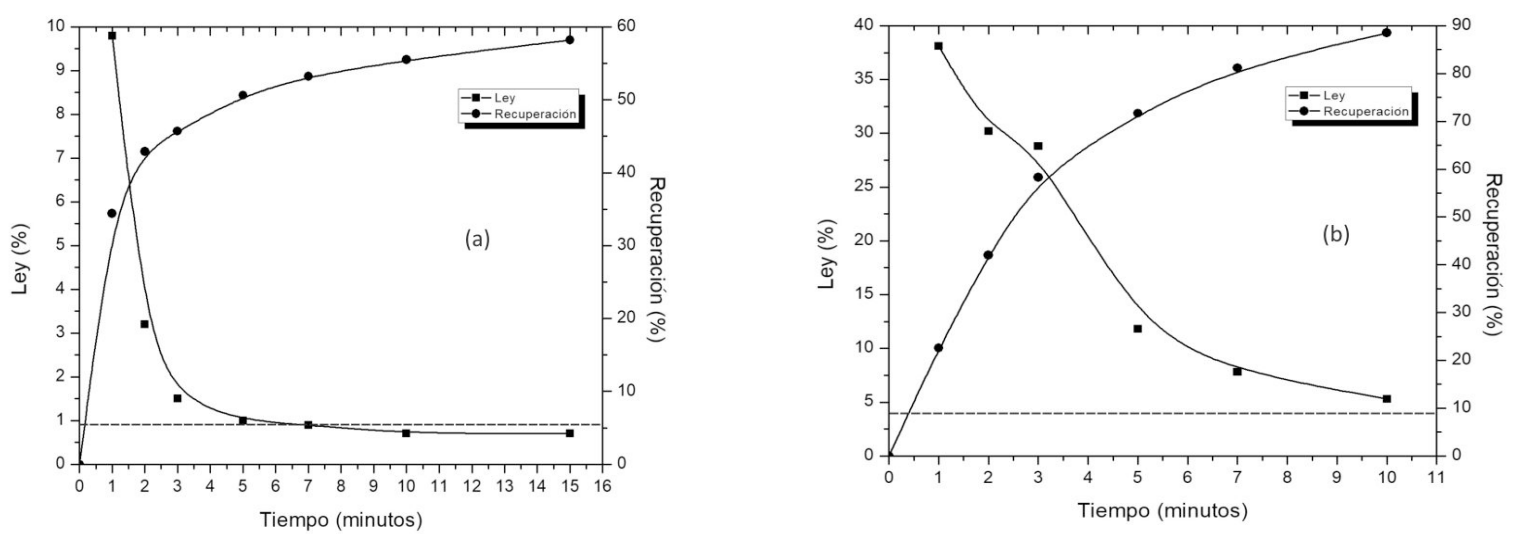

Figura 5. Cinética de flotación rougher (a) y cleaner (b) para escoria de reverbero 
De igual manera, en la Figura 5 (b) se muestra la cinética de flotación cleaner, en la cual se observa que a los 5 minutos de flotación la ley de cobre es de $24,4 \%$, con una recuperación de $71,7 \%$. Tales condiciones experimentales permiten deducir que se requiere incluir una etapa scavenger para incrementar la recuperación.
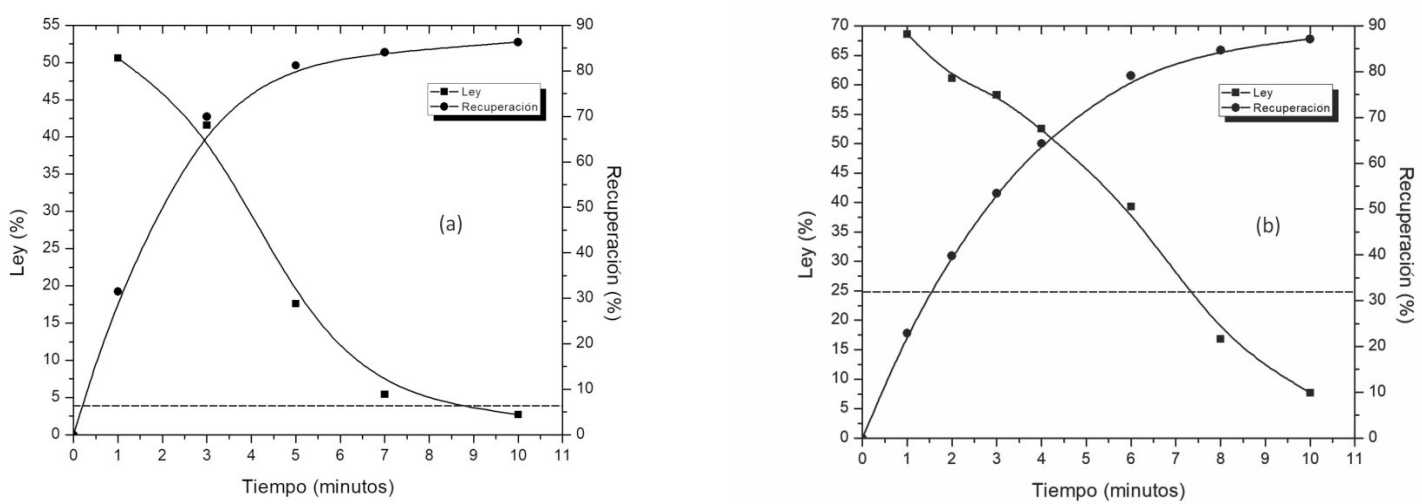

Figura 6. Cinética de flotación rougher (a) y cleaner (b) para la escoria de CT

Las Figuras 6 (a)-(b) presentan la cinética de flotación rougher y cleaner para la escoria de CT. De esta manera, tal como se observa en el gráfico de la cinética rougher, a los 10 minutos de flotación la ley de cobre es de $24,5 \%$ con una recuperación de $86,3 \%$. Aplicando el criterio de Agar, se encuentra que el tiempo óptimo de flotación es de 8 minutos. Similarmente, en la misma figura se muestra la cinética de flotación cleaner, donde se observa que el tiempo óptimo de flotación es de 7 minutos y 30 segundos, con una ley de cobre de 52,7\% y una recuperación de $82,6 \%$.

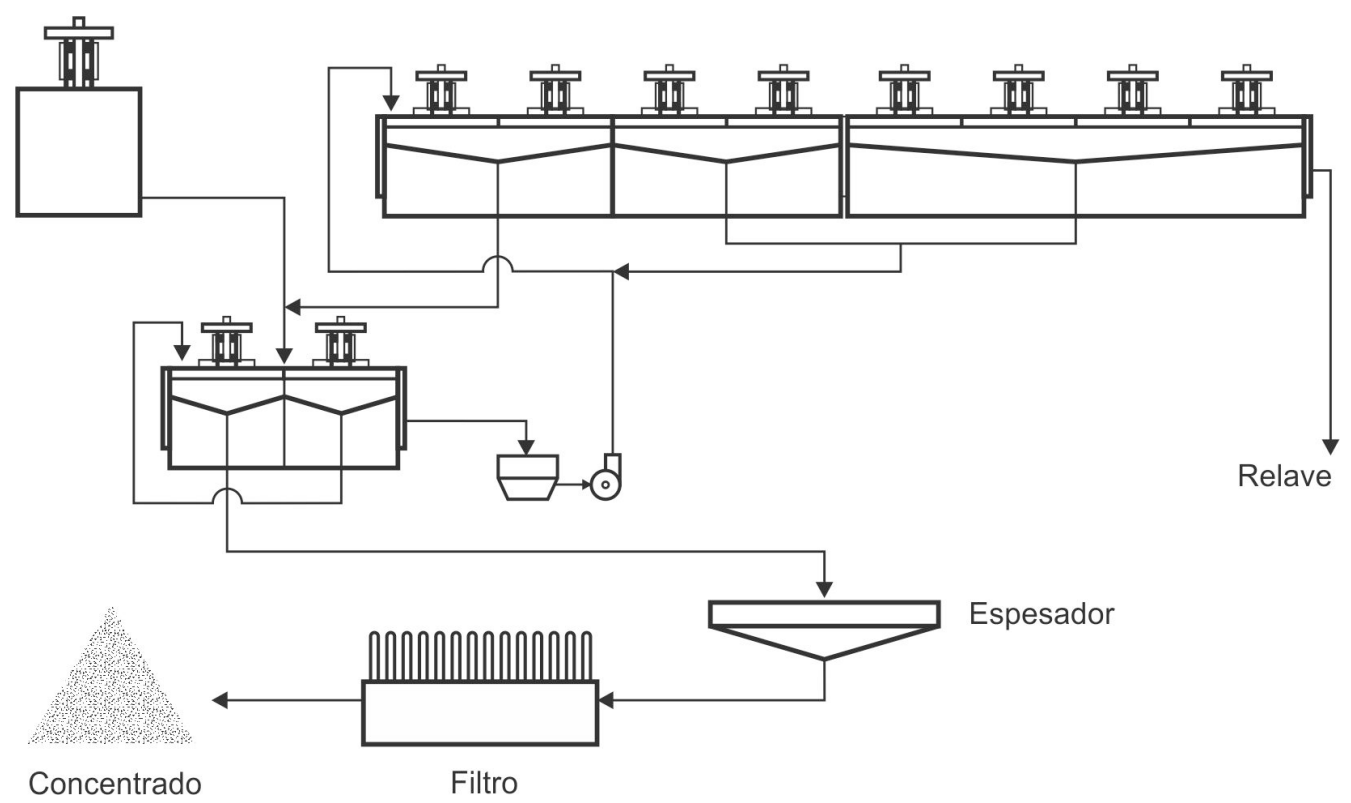

Figura 7. Propuesta para el tratamiento de escoria en planta M. A. Matta

Basándose en las condiciones operacionales a nivel de laboratorio, se propuso el siguiente circuito de flotación para procesar ambos tipos de escorias, tal como se muestra en la Figura 7. 
En base a las condiciones experimentales antes descritas, este circuito de flotación se evaluó a nivel industrial, procesándose 15.615 toneladas de escorias $H R$, obteniéndose un concentrado con $18,2 \%$ de cobre y una recuperación de $56,1 \%$, siendo la ley promedio de la alimentación fue 1,06\% de cobre. De igual modo, se procesó escorias del CT (17.434 toneladas), con una ley de alimentación de 6,4\% de cobre, lográndose un concentrado de $39,1 \%$ y una recuperación de $91,4 \%$. Estos resultados son comparativamente mejores que los obtenidos a nivel de laboratorio, los cuales a su vez son muy satisfactorios. Ello muestra la viabilidad técnica de recuperar cobre a partir de escorias mediante el proceso de flotación, alcanzando resultados similares a los reportados por Guo et al., 2016.

\section{CONCLUSIONES}

Este presente estudio, cuya investigación buscaba mejorar la recuperación de cobre contenido en la escoria del horno reverbero y convertidor Teniente producidos en la Fundición Hernán Videla Lira, de la Empresa Nacional de Minería, permite extraer las siguientes conclusiones:

- La determinación del índice de Bond y de abrasión indicó un valor de 23,7 KWh/t para la escoria del HR y para la escoria del CT un valor de $23,4 \mathrm{KWh} / \mathrm{t}$. El índice de abrasión para la escoria del HR fue de $0,9119 \mathrm{~g} / \mathrm{h}$ y para la escoria del CT fue de $0,7161 \mathrm{~g} / \mathrm{h}$.

- El estudio mostró que la granulometría más óptima para procesar la escoria por flotación tanto para la del horno reverbero y la del convertidor Teniente es de $75 \%$ menor a 75 micrómetros.

- Las pruebas cinéticas mostraron un tiempo óptimo de flotación para la escoria HR para la etapa rougher de 6 minutos 12 segundos y para la etapa cleaner de 5 minutos; para la escoria CT el tiempo óptimo para etapa rougher fue de 10 minutos y para la etapa cleaner de 7 minutos y 30 segundos.

- Los resultados obtenidos a nivel de laboratorio fueron usados a nivel industrial en la Planta M.A. Matta, consiguiéndose resultados satisfactorios. Así, para la escoria HR se logró una ley de $18,2 \%$ de cobre con una recuperación de $56,1 \%$, mientras que para la escoria CT se obtuvo una ley de $39,1 \%$ de cobre con una recuperación de 91,4\%.

\section{AGRADECIMIENTOS}

Para finalizar, los autores agradecen al Departamento de Ingeniería en Metalurgia de la Universidad de Atacama y a Planta Manuel Antonio Matta, de la Empresa Nacional de Minería, por el apoyo económico, técnico y administrativo en el desarrollo del presente trabajo.

\section{REFERENCIAS}

Agar, G.E.; Stratton-Crawley, R.; Bruce, J. (1980). Optimizing the design of flotation circuits. Can Min. Metall. Bull. 73, pp. 173-181.

Alexandrova, R, Paunova, R, Marinov, M and Petrov P. (2014). Study on the behavior of flotation fayalite waste from copper production at nonisothermal heating. Journal of Chemical Technology and Metallurgy, vol. 49, no 1, pp. 90-93. 
Altundogan, H.S., Tumen, F. (1997). Metal recovery from copper $€$ converter slag by roasting with ferric sulphate. Hydrometallurgy 44, pp. 261-267.

Antonijevica, M.M.; Dimitrijevic, M.D.; Stevanovic, Z.O.; Serbula, S.M.; Bogdanovic, G.D. (2008). Investigation of the possibility of copper recovery from the flotation tailings by acid leaching. J. Hazard. Mater. 158, pp. 23-34.

Biswas, A.K., Davenport, W.G. (1980). Extractive Metallurgy of Copper. Pergamon Press, New York, NY, USA.

Bradshaw, D., O'cornor, C.T. (1996). The flotation of low-grade pyrite ores using mixtures of collectors. Hidden Wealth. Johannesburg. Inst. Min. Metall. pp. 89-96.

Bradshaw, D.J., Harris, P.J., O'Connor, C.T. (1998). Synergistic interactions between reagents in sulphide flotation. J. S. Afr. Inst. Min. Metall. 98 (4), pp. 189-194.

Bradshaw, D.J., O'Connor, C.T. (1994). The flotation of pyrite using mixtures of dithiocarbamates and other thiol collectors. Miner. Eng. 7, pp. 681-690.

Bulut, G. (2006). Recovery of copper and cobalt from ancient slag. Waste Manag. Res. 24, pp. 118-124.

Davenport, W.G., King, M., Schlesinger, M., Biswas, A.K. (2002). Extractive Metallurgy of Copper. fourth ed. Kidlington, Oxford, England, Elsevier Science Limited, pp. pp. 57-72.

Demetrio, D., Ahumada, J., Duran, M.A., Mast, E., Rojas, U., Sanhueza, J., Reyes, P., Morales, E. (2000). Slag cleaning: the Chilean copper smelter experience. J. Met. 52(8), pp. 20-25.

Gonzalez, C., Parra, R., Klenovcanova, A., Imris, I., Sanchez, M. (2005). Reduction of Chilean copper slags: a case of waste management project. Scand. J. Metall. 35, pp. 1-7.

Gorai, B.; Jana, R.; Premchand, M. (2003). Characteristics and utilization of copper slag-A review. Resour. Conserv. Recycl. 39, pp. 299-313.

Guo, Z., Zhu, D., Pan, J., Wu, T., Zhang, F. (2016). Improving Beneficiation of Copper and Iron from Copper Slag by Modifying the Molten Copper Slag. Metals 6, 86, pp. 1-17.

Hayes, P.C. (1993). Process Principles in Minerals and Materials Production. Hayes Publishing Company, Brisbane, pp. pp. 173-179.

Herreros, O., Quiroz, R., Manzano, E., Bou, C., Vinals, J. (1998). Copper recovery from reverberatory and flash furnace slags by chlorine leaching. Hydrometallurgy 49, pp. 87-101.

Ip, S.W., Toguri, J.M. (1992). Entrainment behavior of copper and copper matte in copper smelting operations. Met. Trans. B-Process Met., pp. 303-311.

Kim, B. S., Jo, S. K., Shin, D., Lee, J. C., Jeong, S. B. (2013). A physico-chemical separation process for upgrading iron from waste copper slag. Int. J. Miner. Process. 124, pp. 124-127.

Las Fundiciones en la mira. Revista Minería Chilena 405. Marzo (2015).

Mehta, K.D., Pandey, B.D., Permchand, M. (1999). Bio-assisted leaching of copper, nickel and cobalt from converter slag. Mat. Trans. JIM 40, pp. 214-221.

Mihajlović, A., Kamberović, Ž., Korać, M., Gavrilovski, M., Jovanović, N. (2015). The effect of cooling rate of slag from primary copper production in the valorization of copper in the flotation process. Metall. Mater. Eng. Vol 21 (2) 2015 p. pp. 127-141. 
Piret, N. L. (2000). Cleaning copper and Ni/Co slags: the technical, economic, and environmental aspects. J. Met. 52(8), pp. 18-19.

Planta tratamiento de escorias de CODELCO avanza hacia ejecución. Portal minero. Disponible en http://www. portalminero.com/pages/viewpage.action?pageid=112920247.

Rao, G. V., Nayak, B. D. (1992). Flotation of copper from converter slags. J. Mine Met. Fuels 40(34), 131.

Rao, K.H., Forrsberg, K.S.E. (1997). Mixed collector systems in flotation. Int. J. Miner. Process. 51, pp. 67-79.

Roy, S., Datta, A., Rehani, S. (2015). Flotation of copper sulphide from copper smelter slag using multiple collectors and their mixtures. Int. J. Miner. Process 143, pp. 43-49.

Sarrafi, A., Rahmati, B., Hassani, H. R., Shirazi, H. H. A. (2004). Recovery of copper from reverberatory furnace slag by flotation. Miner. Eng. 17, pp. 457-459.

Shen, H. and Forssberg, E. (2003). An overview of recovery of metals from slags. Waste Management. 23: pp. 933-949.

Somerville, M., Norgate T., Jahanshahi, S. (2000). Single Stage Copper Making-Assessment of Slag Treatment Options, Proceedings Minprex 2000, International Conference on Mineral Processing and Extractive Metallurgy, The Australasian Institute of Mining and Metallurgy, Melbourne, pp. 453-459. 